\title{
Determining Habitability in the Milky Way Galaxy
}

\author{
Navya Sahay, Sanah Bhimani
}

\begin{abstract}
Habitability is a new, evolving concept that has captured the interest of many over the past few years. With the advent of new technology, the boundaries of exoplanetary research have been pushed and new insights into the nature of life have been gained. The criteria for what makes a planet habitable and whether the likelihood of life can be predicted is debatable. This research paper aims to connect the stellar and planetary characteristics to their evolution when studying habitability. It aims to create a classification for factors affecting habitability and also presents a guiding framework that can be used to determine the habitability of the planet. The paper connects the framework to pre-existing knowledge and data about habitability derived from Venus, the evolution of life on Earth, and Kepler 452-b.
\end{abstract}

Index Terms - Physics and Astronomy, Astronomy and Cosmology, Habitability, Exoplanets.

\section{INTRODUCTION}

"Are we alone in the universe?". Through the years, there have been several researchers who have attempted to answer this question. As of now, the question remains unanswered because the only example of life that we possess is the one currently residing on Earth itself. However, the likelihood of life on other planets can be, to an extent, predicted and classified through the concept of habitability.

Habitability is often defined as the ability to support life on a planetary surface. Habitability is decided by a variety of factors linked to stellar and planetary characteristics. These are not only indicative of the probability of life on a planet, but also help determine a "habitable zone" of a certain star. The "habitable zone" (HZ) is the "circumstellar region where a terrestrial radius and mass planet can maintain liquid water on its surface" [15]. An area of certain orbital distances around a star where life is most likely to occur makes the habitable zone. The boundaries of the $\mathrm{HZ}$ are controlled by stellar and planetary features such as type of star, stellar flux and climatic conditions. Each habitable zone has an inner edge (Inner Habitable Zone or IHZ) and an outer edge (Outer Habitable Zone or OHZ). The edges of the zone are determined by the maximum or minimum greenhouse limits (limit of the greenhouse effect warming the planet) that a planet can have to sustain life. Runaway and moist greenhouse limits also come into play here as discussed in further sections. The habitable zone differs for different types of stars and planets as shown in Figure 1.

Navya Sahay, High-school student, educated at Neev Academy. Sanah Bhimani, Yale Ph.D Candidate

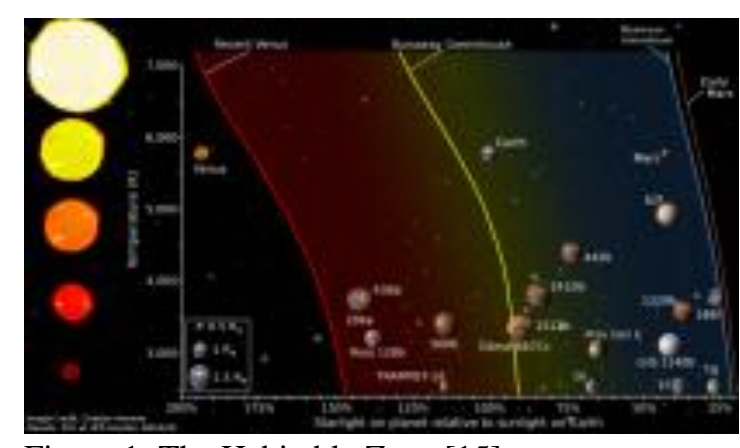

Figure 1: The Habitable Zone [15]

The habitability of a planet can be decided both qualitatively and quantitatively. The next section deals with a quantitative measure of habitability.

\section{DRAKE EQUATION: MEASURING HABITABILITY}

The Drake Equation is a probability theorem used to approximate the number of extra-terrestrial civilizations in the Milky Way Galaxy. Formulated by Frank Drake in 1961, it serves as a tool for approximating the likelihood of intelligent life in the universe [20]. There is another version of the equation developed by Professor Sara Seager, who altered the equation so that it calculated the number of habitable planets in the galaxy [17]. These two equations serve as the inspiration for this paper. However, the paper does not take a quantity-based approach towards habitability, rather focusing on the factors like stellar luminosity, planetary rotation, etc that can influence its habitability and using case studies to illustrate how life can occur as a phenomenon.

\section{STELLAR COMPOSITION AND EVOLUTION IMPACTING HABITABILITY}

In the search for candidates for habitability, the host stars are one of the first places to start as their characteristics are integral to the evolution and sustenance of life.

\section{A. Stellar Characteristics That Determine Habitability}

There are several characteristics of a star that influence the probability of habitability. Some of the factors are stellar spectral energy distribution, stellar winds, stellar mass, luminosity, age, X-ray/UV emission, magnetic field, and stellar multiplicity [15]. This paper will focus on stellar mass, luminosity, stellar winds, emissions, and age. Most of these 
factors are not arbitrary as they are often dependent on the mass or type of star. Hence, when exploring habitability, these are some of the first characteristics to keep in mind as they can narrow down the search.

\section{B. Stellar Evolution: Mass, Luminosity And Lifecycle/Age Of A Star}

The luminosity and mass of a star are linked, especially with respect to stellar evolution as there are certain stages in a star's life cycle that are more suited for life than others. Stellar luminosity affects the amount of energy that a planet receives from its host star, which in turn determines the width of the habitable zone of the planet around the star. The habitability limits are determined by stellar radiation: one limit is the moist greenhouse limit where the stellar radiation is sufficient to warm the atmosphere causing a specific water vapor volume ratio and the other runaway greenhouse limit is when the surface water is vaporized [15]. M-dwarfs have low luminosity and have longer life cycles, unlike high mass stars. This is beneficial as a long life cycle gives time for atmospheric evolution that is needed to sustain life to take place.

\section{Stellar Abundance/Metallicity and Age}

The chemical composition of stars is crucial in determining their age. The nuclear fusion of elements heavier than hydrogen and helium in the star and the ratios of such elements in the star determine its age and the insolation a planet would receive from the star at various orbit distances. This in turn helps mark the habitable zone. Additionally, chemical compositional data can be used to predict how long the planet will remain in the habitable zone (it should ideally remain there for at least 2 Gyr) [11]. If the star has already proceeded to the fusion of heavier elements or the abundance of elements is indicative of stellar radiation that does not support environmental sustainability, then the planet may be unable to sustain life for a long period as the star has almost reached the end of its life or nuclear fusion.

\section{Activity of the Star: Stellar Winds and Radiation}

Stellar activity, while not an arbitrary factor, does determine whether life can be sustained on a planet. Hence, even if life is established, high stellar activity from a violent star can demolish any surface life that managed to evolve [11] The level of stellar activity is dependent on sunspots, flares, $\mathrm{UV}$ and X-ray radiation, and coronal mass ejections. The sunspots and flares are a result of the magnetic field interactions of the star's convection zone (where plasma movements and convection currents occur) ("Sunspot"). The depth of the convection zone of a star is decided by its mass. High mass stars have too shallow a zone to generate muchmagnetic energy. Low mass stars, on the other hand, have high stellar activity due to a deep convection zone.

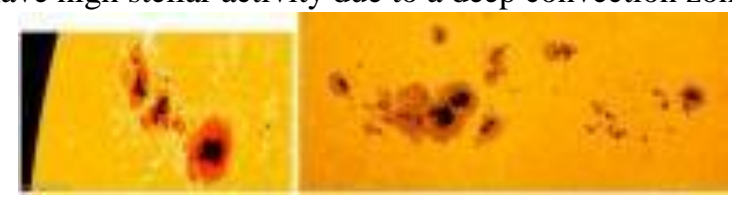

Figure 2: Sunspots [27]
The generation of a magnetic field is also caused by the rotation rate of a star, which varies throughout its age. Younger stars tend to have a higher rotation rate and thus more magnetic activity than older stars. Hence, the age and mass of the star must be considered while observing its magnetic activity levels. Unless the star is "quiet", such activities could severely impact the physical characteristics of exoplanets. In fact, there was a study conducted on the dwarf star TRAPPIST-1, which showed how the planets surrounding it underwent atmospheric loss due to its stellar winds [5] . Additionally, UV and X-Ray radiation of a star must be held in check as they can cause ozone layer depletion and atmospheric loss.

Thus, the stellar mass, type, and age are crucial factors through which other factors like stellar abundance and activity can also be determined. Observation of these fixed, basic factors and the correlated characteristics can lead to the identification of star candidates suitable for life.

\section{E. Ideal for Habitability: Goldilocks Stars}

After delving into stellar characteristics that may impact habitability, the ideal type of stellar characteristics that would increase the chances of habitability should be studied. Goldilocks stars are a certain type of stars that are just right to foster life. They are not too hot, nor too cool, and not too violent. Contrary to common misconceptions, Goldilocks stars are not necessarily like our Sun- a G-type star. Other than G-type stars, the smaller and more abundant M-type dwarfs are also not ideal for habitability. M-type stars are too active and could lead to extreme environments that may not be able to host life [22]. They have too much energy when they are younger, which could boil oceans off of a habitable planet, removing the source essential for life: water. Also due to their size, these red dwarfs have narrow habitable zones that are too close to the star, rendering the planet open to harmful UV/X-ray radiation exposure.

Recent studies and discoveries point to K-type stars being the most ideal for habitability [9]. K stars are dimmer than the sun but brighter than M-type red dwarfs. They can live for about 17 to 70 billion years, which is much longer than the sun-like stars, giving planets more time to evolve. They also have fewer solar flares when they are younger, so pose less of a threat to destroy life. K-stars are not only ideal but easier to detect. There are 3 times more K-type stars than G-type stars in our galaxy, making explanatory research for terrestrial planets around them also easier.

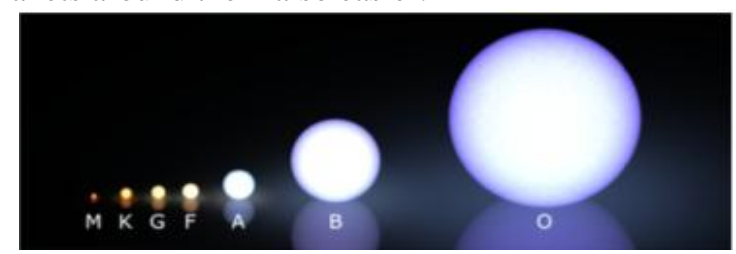

Figure 3: Types of Stars (EarthSky)

\section{PLANETARY CHARACTERISTICS: FORMATION AND EVOLUTION}

Once the host stars are deemed suitable, the search for the ideal host planet begins. 


\section{A. Planet Type And Mass}

There are a variety of exoplanets formed, differing in mass, density, and size. Hot Jupiters, super-Earths, mini Neptunes, and ice Giants are just some of the types classified by scientists.

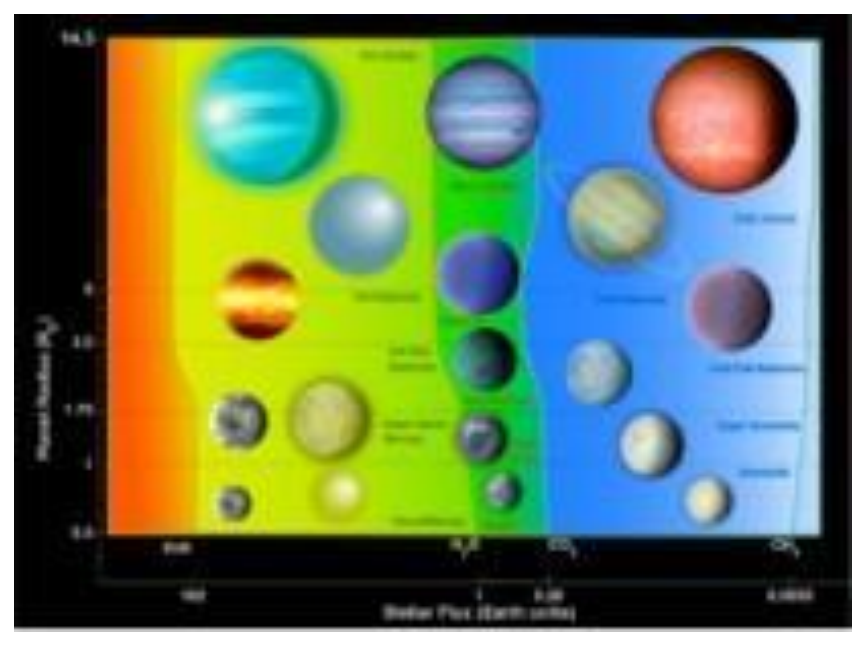

Figure 4: Types of Planets [15]

If exoplanets are viewed with the lens of habitability, however, only a few fit the criteria. Focusing on terrestrial words that have a radius of no more than $1.5 \mathrm{R}$ certainly narrows down the search [15]. Typically, exoplanets around this radius have densities closer to rock and metal. It should be noted that sub-Neptunes, a type of exoplanets that are smaller than Neptune and bigger than Earth, show extreme promise [15]. Sub-Neptunes are the most commonly detected, occupying a large fraction of the known exoplanets. They can be further divided into two categories: mini Neptunes that have mainly an icy composition and the more rocky super-Earths. Super-Earths are terrestrial and have densities higher than those found in the solar system. Unfortunately, finer nuances about their properties are yet to be detected as the equipment currently available is not sensitive enough [15] However, they are certainly possible candidates for life, considering their radius and composition. While some of the planets discovered may have similarities with Earth, they are nevertheless often different from the properties of planets in our solar system. Hence, it is important to note that the criteria for habitable exoplanets may vary and is flexible, subject to any new knowledge or insights gained from ongoing investigations.

Additionally, the mass of a planet as a physical characteristic has a large impact on the habitability of a planet The mass and the radius of the planet often determine its atmospheric density and evolution. During a planet's formation, a planet's mass results in gravitational compression and the dispersal of its protoplanetary disk which reduces the pressure and causes atmospheric mass loss [21].

As the core of the planet cools, the atmosphere shrinks to a certain thickness. The final thickness of the atmosphere is determined by the mass of the planet and its heat capacity. It is important for the planet to have a certain level of atmospheric thickness in order for temperate climates with the condensation of $\mathrm{H} 2 \mathrm{O}$ and formation of carbon dioxide clouds to be possible [15]. Such processes are integral to life. For instance, while Mars lies in the Habitable zone, due to its low mass (0.1 Earths), it does not have a large enough atmosphere to support life [15].

The mass and the radius of the planet are standard factors that are easy to measure via the transit method. They are the first-order or primary characteristics to account for when looking for life. They are often determined by stellar and planetary evolution and hence are not extremely unpredictable or arbitrary.

\section{B. Planetary Rotation Rate, Eccentricity, And Obliquity}

Factors that are decidedly arbitrary include planetary rotation, eccentricity, and obliquity. Yet, despite being subject to change, these factors are responsible for key atmospheric and climatic phenomena [15]. They determine the stellar radiation received by a planet at a particular point in time. Obliquity is a factor responsible for the seasons that take place on Earth [10]. A certain level of obliquity is needed to maintain seasons and atmospheric processes. However, high obliquity planets may suffer from extreme seasonal variability and climate instability (Glister). Moreover, the rotation rate of a planet determines the Coriolis force and atmosphere circulation of a planet [1].

These factors are all interrelated. For example, if a planet has low orbital eccentricity, then it will have a low rotational rate. Eventually, such a planet may become tidally locked. Tidal locking is a result of gravitational interactions between the host star and its planet that lead to synchronous rotationsonly one side of the planet facing the star at all times [15]. This can cause a planet to face extreme temperatures and even atmospheric collapse. It is a feature that is certainly not desirable. Unfortunately, planets in the habitable zone and relatively close to the star are more likely to become tidally locked, especially if they are orbiting MGK dwarf stars [1]. The tidal lock radius of a planet is determined by the mass of a star, the mass of a planet, the presence of a satellite, heat dissipation, and more. Whether a planet is tidally locked or can become so is subject to change, dependent on the planet's individual history of formation and gravitational interactions.

Tidal obliquity and eccentricity may also change due to factors such as gravitational interactions with neighbouring celestial bodies. Certain levels of eccentricity are expected in certain stages of a planet's revolution cycle (Kopparapu et al. 9-11). For instance, when the core is cooling, if the eccentricity is maintained, then the planet's core would solidify, exposing it to harmful stellar erosion. Hence, these factors are second-order and arbitrary, dependent on a range of first-order factors and chance. There are other factors like geological activity, tidal effects, and magnetic fields that also impact the habitability of a planet.

\section{Atmosphere And Climate}

Atmosphere refers to a mixture or layer of gases surrounding the planet. Atmospheric retention is dependent on the gravity of the planet and hence, the existence of an atmosphere can be considered as a fixed or non-arbitrary 
factor. The atmosphere is crucial for habitability because without atmospheric pressure, any existing water would evaporate or sublimate, robbing the planet of any pre-existing potential for life that it may have had. The atmosphere cannot be too shallow, especially in synchronously rotating planets where temperatures can vary and result in atmospheric collapse [15]. Nor can it be so dense as to block any stellar radiation incoming.

Other than water retention, an atmosphere also helps maintain the thermal equilibrium of a planet by striking a balance between stellar radiation and the planet's own thermal radiation. Gases like carbon dioxide are essential for the greenhouse effect needed to maintain surface temperatures on a planet. Therefore the gas cycles on a planet such as a silicate weathering cycle that aids the carbon cycle are the chief factors contributing to thermal stability (Kopparapu et al. 13-14). Such cycles are a product of different complex tectonic and magnetic systems interacting, hence their existence is an arbitrary factor. The atmospheric composition also plays a part in ensuring the sustenance of life. For instance on Earth, the infamous ozone layer protects life from the harmful effects of UV light [15].

Atmospheric evolution is another aspect that one must consider. Like the terrestrial planets of our Solar System, a planet's loss of its primordial $\mathrm{H} 2$ atmosphere through outgassing is beneficial as otherwise it may end up like the Gas giants of the Solar System with a dense and opaque atmosphere, unsuitable for life. However, merely developing a secondary atmosphere is, unfortunately, not enough. Mars and Venus (see section 6.1) are prime examples of how secondary atmospheric evolution can go wrong. Mars has been stripped of its atmosphere, rendering it unable to maintain a warm climate [15]. Venus, while having retained an atmosphere, has lost its water to space. The runaway greenhouse effect on Venus that caused this ocean loss is a constituent that threatens habitability. Atmospheric escape is dependent on a multitude of factors. Some such as the stellar wind and activity, UV radiation, mass and magnetic field of the planet are definitive. M-dwarfs have high activity phases that can strip a planet, especially one of low mass and weak magnetic field, of its atmosphere [22]. Other factors like impact collisions are arbitrary. However, the type of star and planet definitely is indicative of whether the said planet is vulnerable to atmospheric loss. A complete loss of an atmosphere only results in extreme climatic conditions that are not suited for life. Hence, the photochemistry and gravitational interactions of the planet impact its atmospheric evolution and sustenance.

\section{Galactical Effects on Habitability}

The environment a planet exists in its locality and neighbourhood, and its proximity to other celestial bodies can have a strong impact on its potential for habitability. For instance, the Earth's habitability is strongly dependent on its plate tectonics which spurs its geological activity and is a result of nearby supernovae [13]. The heat that generates these plate tectonics is believed to be from the decay of uranium 235 and 238 and thorium 232 and potassium 40. The supply of unstable isotopes is believed to come from type 2 supernovae. This could also be because of neutron star mergers.

While supernovae can be beneficial to habitability in some ways, their vicinity to the planet in question can have dangerous implications as well. High energy supernovae for instance can impact the earth's biosphere by depleting its ozone layers [13]. Additionally, gamma-ray bursts are another galactic occurrence that can have detrimental effects on life as their harmful nature can lead to mass extinction. Overall, galactical effects, while important to consider when looking for the ideal planet, are arbitrary and unpredictable. The proximity to a supernova or some other galactical occurrence is often based on forces of chance.

\section{FRAMEWORK}

To summarize the above findings, a guiding framework of factors that help determine habitability is appropriate. The framework is presented below as a flowchart. The factors are categorized as first order and second order. First-order factors as aforementioned are the primary characteristics that determine habitability. They impact the probability of life's very existence. The second-order factors are those that are more due to a combination of first-order factors and may not just impact the existence of life, but its sustenance and longevity. For example, stellar activity not only impacts the existence of life, but high levels of it can cause any pre-existing life to become extinct. Hence, it's a second-order factor. Further categorization of the factors has been done in terms of whether they are definitive or arbitrary. Definitive factors are based on pre-conditioned processes that can be predicted to an extent, while arbitrary factors are those that depend more on the forces of chance and are unpredictable.

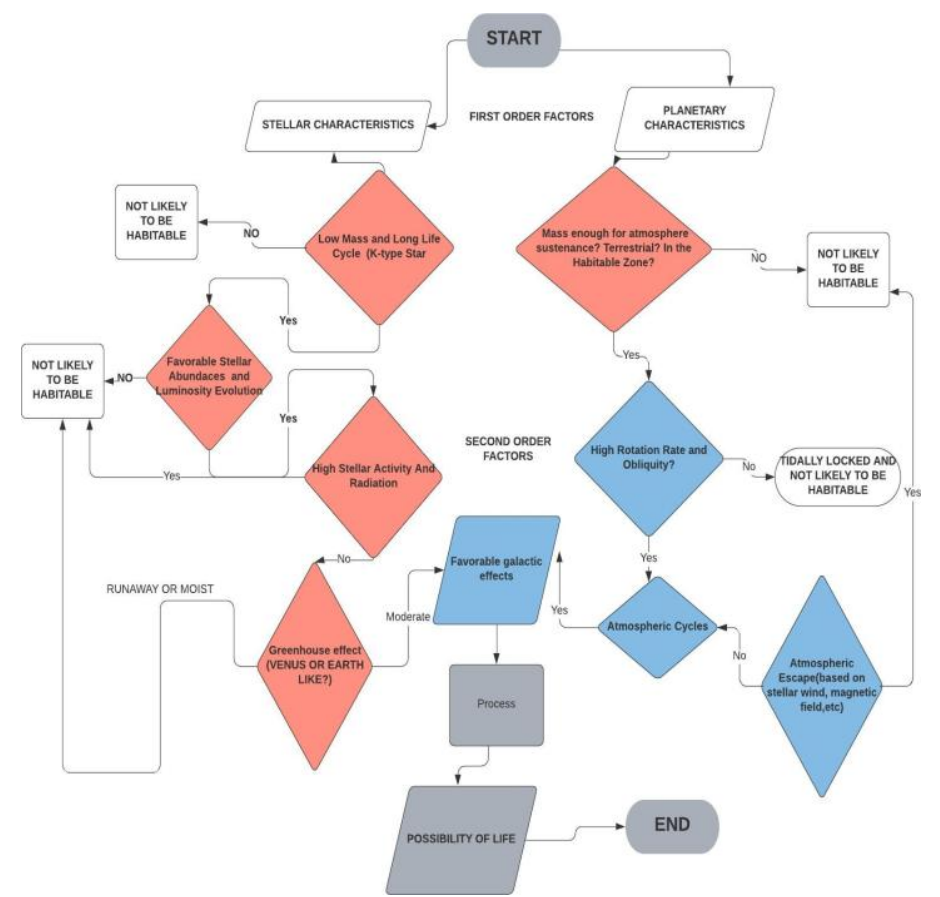

Figure 5: Framework of factors determining Habitability

The next section of the paper puts the theoretical framework above into a more practical perspective by exploring different case studies and scientific data collected 
about potential candidates for habitability. These case studies are meant to illustrate how the framework for habitability needs to be flexible as it may change based on new discoveries. Also, the case studies help illuminate some of the characteristics found in the framework, by offering real-life examples and processes.

\section{CASE STUDIES OF THE SOLAR SYSTEM}

\section{A. Venus Case Study}

Often named as Earth's twin, Venus has remained an area of interest for years due to its similarity to Earth and the popular hypothesis that it was once possibly habitable. Through climate models and current characteristics of Venus, it has been inferred that when the sun was dimmer, Venus could have been in the inner edge of the Habitable Zone and contained water. However, due to the successive effects of a variety of factors such as the excessive amount of carbon dioxide on Venus, the stellar winds of the sun and the runaway greenhouse effect, Venus lost most of its ocean to space and is now the hottest planet in the Solar System with huge clouds of sulfuric dioxide [15]. Its current state is the polar opposite of being habitable.

Venus and Earth's early evolution may have been similar because they were provided with roughly the same amount of volatiles from the solar nebula that formed the Solar System [3]. However, at some point, their evolution diverged due to four main points of differences between them. Firstly, Venus did not have a large reservoir of water like Earth. It may have had water once, but even that was lost to space. Second, Venus's plate tectonics were stagnant, unlike the dynamic plate tectonics on Earth that contribute to a number of climatic processes and help maintain atmospheric equilibrium [6]. Third, Venus did not have a magnetic field like Earth, a feature that would only exacerbate its runaway greenhouse effect. Finally, Venus is much closer to the Sun than the Earth and the extra insolation it received as a result also caused the runaway greenhouse effect. These four features of Venus interacted with each other to reduce Venus's potential for habitability.

\section{a. Solar Insolation And Hydrogen Escape}

The hypothesis that Venus may have had water in the past is heavily based on the evidence of large $\mathrm{D} / \mathrm{H}$ ratios found on Venus that indicate that it may have lost its hydrogen and oceans at a particular period of its history. A primary cause for this was theclose proximity early Venus had to the Sun. After the accretion process, Earth's critical temperature is estimated to be 600 Kelvin which was lesser than the critical temperature that could lead to the runaway greenhouse effect [3]. Hence, water was able to condense on Earth. However, on Venus, due to the solar flux it was subjected to, the surface temperature was 700 Kelvin, which was more than the critical temperature, thereby preventing water from condensing.

This shows the fragility of a planet's habitability and how easily potentially habitable planets can suffer from the runaway greenhouse effect. The "Venus Zone" is a term that refers to the runaway greenhouse limit in which planets can potentially yield Venus-like surface conditions as shown in the diagram below (Kane).

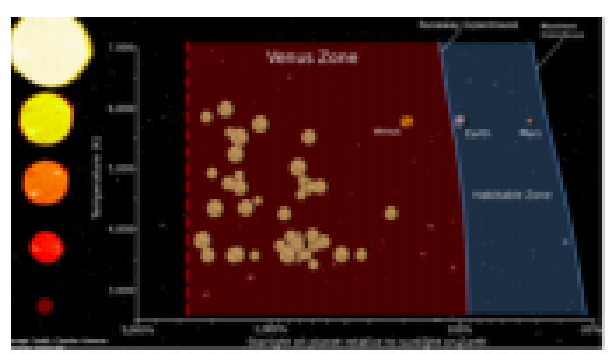

Figure 6: Venus Zone(Kane)

Any planet in the Venus Zone of their particular host star can deviate from the expected evolution due to the apotheosis of solar influx and water loss.

\section{b. Plate Tectonics}

Part of the reason why Earth is able to host life is due to the carbon cycles on Earth that maintain atmospheric temperatures and greenhouse stability. These carbon cycles are often controlled through degassing of $\mathrm{CO} 2$ into the atmosphere, weathering of silicate rocks that serve as a sink for carbon dioxide, and oceans that also observe carbon dioxide [6]. All these carbon sinks prevent the runaway greenhouse effect from taking place. The weathering rate on the planet bears a strong correlation with the velocity of its plate tectonics. Hence, on Venus, due to virtually stagnant plate tectonics, the conditions for a runaway greenhouse effect were favourable and possibly contributed to its water loss and current hot climate. Venus's weak carbon cycle and slow plate tectonic speed may have been one reason why its evolution did not mirror Earth's (Driscoll).

\section{c. Water Loss Magnetic Field}

Despite the runaway greenhouse effect occurring, Venus could still have retained some of its water had it had a strong magnetic field. A strong magnetic moment could have offset and balanced the solar winds at the top of the atmosphere, allowing the planet to retain some of its water vapor [6]. Even then the water would have been in gas form, but still, the planet may not have been as dry as it is now.

It must be noted that the above section is based on modelling and the hypothesis of researchers. Venus's evolution still remains largely a mystery as there is comparatively less physical, concrete evidence to substantially conclude any of these theories. Nevertheless, Venus remains an integral part of exoplanetary research because it demonstrates how habitability is dependent on a variety of factors, and a small deviation from one of them can cause extremely different conditions from those expected. Venus should therefore be taken as a cautionary tale to view potential candidates for habitability as some may seem very similar to Earth, but may in fact be closer to Venus's conditions. A lot of planets may appear to have favourable conditions but may suffer from a fate, not unlike Venus.

\section{B. Earth Case Study}

When studying habitability, delving into the topic of Earth 
is inevitable as it is currently the only known example of advanced life. By studying how life evolved on earth, insights into the possible criteria for habitability can be gained.

\section{a. Formation Of The Solar System and Planetary Accretion}

The formation of the Solar System started with the collapse of a giant molecular cloud, which led to large dense cores being formed. One of the cores formed the solar system. Studying meteorites gives a hint of the first solid material formed in the pre-solid nebula [21]. A shock wave from a supernova may have triggered the formation of the sun as it would have caused denser regions to collapse. The sun may have interacted with other stars which would have caused the material to cluster and form the anomalous objects orbiting the sun in the solar system. Due to the conservation of angular momentum, the nebula continued to spin faster during its collapse. As the materials condensed, the atoms collided, causing an increase in heat, especially in the centre. Gravity, magnetic fields, pressure, etc caused the nebula to flatten out and create a protoplanetary disk and a protostar [21]. The planets were thought to be formed from the solar nebula left after the sun's formation. Planets began as dust grains that formed clumps and eventually planets through accretion. The cosmic dust collided with itself due to gravity to form planets. The inner solar system or rocky planets are believed to be formed by metallic compounds of high melting points. The inner solar system was too warm for volatile molecules like methane and water to condense [21].

It took years for planets like the Earth to form via accretion. The process of accretion caused the Earth to develop large amounts of heat that formed magma oceans in its interior and volcanic eruptions as a result of large bodies colliding with it [21]. Accretion also led to the formation of the core and the subsequent magnetic field. This theory has been substantiated by evidence gained from the Apollo mission's insights into the moon as well as the study of isotopes found on Earth [18]. If a planet's core formation and magnetic field is dependent on accretion, then it is not surprising that habitability can often be random and unpredictable because a lot of the features of Earth were dependent on the chance collisions that it was part of. This Hadean Earth as it is called, which existed about 4.5 billion years ago, bore large similarities to Venus. After this, the Earth began to cool in the Archean period that was between 4.5 to 2.5 billion years ago [23].

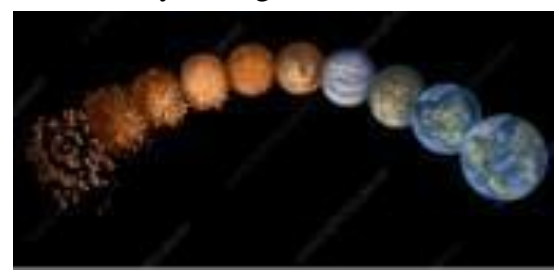

Figure 7: Formation of the Earth [16]

\section{b. Development of Atmosphere and Life}

The atmosphere of the Earth formed due to the outgassing of the mantle. By observing isotopes, geochemists have concluded that about $80-85 \%$ of the earth's atmosphere is outgassed in the first million years, while the rest was slowly outgassed over the next 4.4 billion years [17]. Earth's primitive atmosphere was very like Venus, except for the significant difference of containing water. It is thought to have been dominated by high amounts of carbon dioxide and nitrogen, along with methane, ammonia, and sulphur dioxide. There is much debate about how the carbon dioxide, if it had been so prevalent in the early atmosphere, was removed and buried in carbonate rocks. Some argue that the carbon dioxide was removed through geological activity, while others favour the Gaia hypothesis of early life (like phytoplankton) absorbing the carbon dioxide from the atmosphere [18] Whichever the case, Earth's fate was different from Venus precisely because a majority of the carbon was removed from the atmosphere.

There is little consensus as to when life first appeared on Earth. While some evidence can be gathered from the isotopes found in the rocks on Earth to chart the history of the planet, when exactly life began remains debatable. There is evidence of blue-green algae existing as far back as 3.5 billion years ago, however, more concrete proofs are needed to back the theories proposed [18]. Nevertheless, even our incomplete knowledge of Earth provides insights into how life is formed: through accretion that creates a core, magnetic field, and plate tectonics and through the development of a feedback loop that ensures the stable exchange of gases between the atmosphere and planet. A lot of chance factors may have worked in each other's favor to create life as we know it on Earth, but these key features are definitely essential to habitability.

\section{Kepler 452-b Case Study}

When looking outside our Solar System, this planet is the first that comes to mind when thinking of habitability.

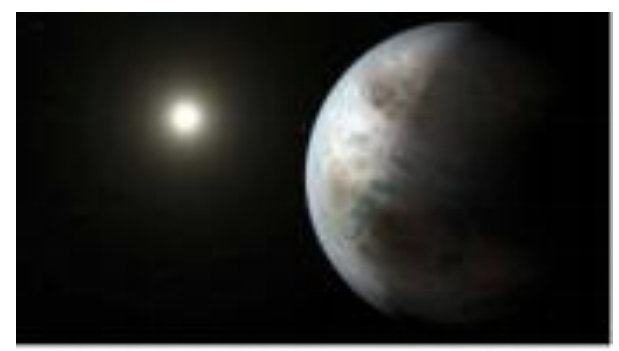

Figure 8: Kepler 452b [25]

Often called Earth's cousin or Earth-2.0, Kepler 452-b is one of the strongest candidates for habitability. However, due to the limitations of technology, there are several unknowns revolving around this Earth-like planet and its potential for habitability. For instance, because the star for Kepler 452-b is 1.5 billion years older than the sun, it is at a later stage of its stellar cycle where its luminosity is higher, putting it in possible danger of the runaway greenhouse effect that may have already taken place or is likely to do so [24]. Even the strongest candidate for habitability may fall down a Venus-like path.

Despite such predictions, there are some qualities of this exoplanet that point favourably to habitability. It is estimated to be $60 \%$ wider than earth in terms of diameter and has a 
strong chance of being rocky or terrestrial [18]. Its period is 385 days, extremely close to our own [12]. It is $5 \%$ further away from its star than the Earth is from the sun [25]. This feature could work as a benefit as it may counteract the extra luminosity it receives from the older star. But these are mere speculations as there is too little data available and indeed some question if the planet even exists due to the high error levels in such a field (discussed further in Section 7).

An interesting feature of Kepler $452 \mathrm{~b}$ is that it has existed for 6 billion years in the habitable zone of its star, much longer than Earth, giving possible life time to evolve. Although there is uncertainty in the probability of habitability on this exoplanet, Kepler $452 \mathrm{~b}$ is a useful case study to use to develop our criteria for habitability. For instance, not only is the age of the star important, but the age of the planet and how long it has been in the habitable zone can also be a deciding factor.

\section{METHODS OF DETECTION}

While this paper mainly explores the theoretical aspects of habitability, such theoretical conclusions are only made as a result of the data collection linked to exoplanets. This section aims to highlight some of the ways exoplanets are detected.

\section{A. Types Of Methods Of Detecting Exoplanets}

There are several methods of detecting exoplanets. Usually, a planet's size, mass, and radius are indirectly measured because a planet is often overshadowed by its host star and it's difficult to obtain direct images of the said planet. One method of detection is the radial velocity method in which the difference of a star's rotation due to the planet's gravity is measured [26]. A planet's gravity causes variation in the star's speed as the star moves towards or away from Earth. Such deviations confirm the presence of a planet and also helps approximate the mass of the said planet. The radial velocity method is most effective for low-mass stars as they are more likely to have visible effects from the planet's gravity.

Another method of detection is the transit photometry method. This method provides insights about a planet's radius. When a planet transits across its host star, a minute drop in the brightness of the star is observed which is used to estimate the planet's size and radius in relation to the size of its host [26]. A light curve's depth is used to illustrate the radius of the exoplanet. However, this method requires perfect alignment with the star and astronomer [26]. Also, the method is vulnerable to false detections especially due to eclipsing binary systems which would create their own transits and interfere with the photometry as explored further in the next section.

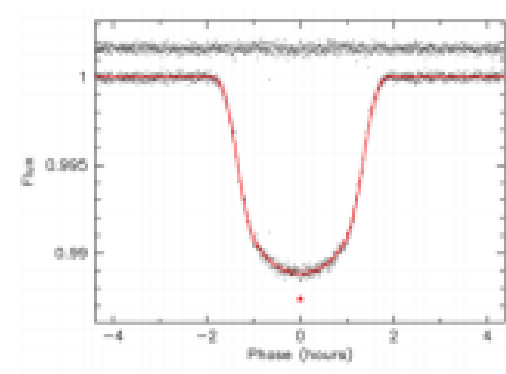

Figure 9: Light Curve [26].

\section{B. Transit Photometry Used To Detect Kepler 452b}

This section will be on the method of detecting exoplanets, namely Kepler-452b, a candidate for habitability, to show the level of precision needed in exoplanetary detection.

Kepler 452b was discovered by NASA's Kepler spacecraft. Initially, this planet's transit signature was not detected by the initial code- SOC.9.1 developed. This is because the code in question was made intentionally very aggressive to block out any possible noise that may interfere with the findings. A second, less aggressive code- SOC.9.2 was later developed which identified Kepler 452b's transit signature [8].

After identification, the team of researchers also conducted a set of data validation tests as there are many celestial bodies that could have interfered or been the source of the signatures. To ensure that every next transit, after one period, on the light curve matched with the others and the signature was of Kepler $452 \mathrm{~b}$ only, an odd-even data validation test was conducted. In this test, the odd transit signatures (first dip, third dip, fifth dip, etc.) and the even transit signatures (second dip, fourth dip, sixth dip, etc) are plotted separately to see if they all match with each other [8]. Then, the two graphs are compared to each other and if they match, there is only one transit signature, that of the desired planet. In the case of Kepler 452b, the transit signatures did match (as shown in the image below), validating the data and reducing errors.

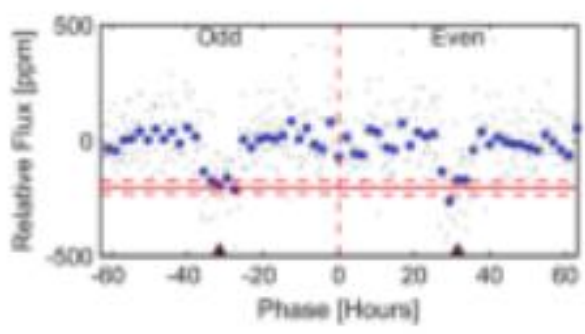

Figure 10: Odd Even Test [8]

Such level of precision and data validation is needed when doing exoplanetary research and is the reason why the quest for habitability is a long one that has only just started.

\section{CONCLUSION}

In conclusion, habitability as a concept is still evolving as we keep gaining new insights into its nature through recent exoplanetary research. The case studies of Venus and Earth along with the discovery of Kepler $452 \mathrm{~b}$ especially inform 


\section{Determining Habitability in the Milky Way Galaxy}

this conclusion as they show possible changes and second-order and/or arbitrary factors one must consider if a candidate already has the basic properties needed to host life. For instance, as seen in the framework, there is a factor of the greenhouse effect to observe if a planet is more likely to develop Venus-like or Earth-like conditions. The criteria or method to predict habitability will continue to change. As the Methods of Detection section demonstrated, exoplanet discoveries require immense precision and the data collected must account for some level of uncertainty. Hence, as a result, the very definition of habitability continues to develop as more data is gained. However, some aspects such as the first-order factors or the fact that there are some parts of habitability that are based on chance can be safely accepted as definitive. Therefore, as scientists continue to examine and explore the universe, looking for signs of life, they must consider these factors of planetary and stellar characteristics that impact the probability of habitability.

\section{REFERENCES}

Barnes, Rory. Tidal Locking of Habitable Exoplanets, 2017.

[2] Briggs, Andy, "What Are Exoplanets?" EarthSky, earthsky.org/astronomy essentials/what-are-exoplanets: $\quad$ :text=Exoplanets\%20are\%20 planets\%20that $\% 20 \quad$ orbit, $1 \% 2 \mathrm{C} 000 \% 20 \quad$ more\%20 more\%20awaiting\%20confirmation.

[3]Chassefiere, Eric et al, "Early Evolution of Venus' Atmosphere and Hypothesized Formation of a Transient Water Ocean."

[4] The Evolution of Venus: Present State of Knowledge and Future Exploration, 2011, doi:https://doi.org/10.1016/j.pss.2011.04.007.

[5] Dong, Chuanfei. Atmospheric Escape from the TRAPPIST-1 Planets and Implications for Habitability, 28 Dec. 2017.

[6] Driscoll, P., and D. Bercovici. "Divergent Evolution of Earth and Venus: Influence of Degassing, Tectonics, and Magnetic Fields."

[7]NASA/ADS, ui.adsabs.harvard.edu/abs/2013Icar..226.1447D/abstract.

[8]Ehrenpreis, Michelle. "Discovery." DISCOVERY AND

VALIDATION OF $^{\circ}$ Kepler-452b: A 1.6 R A SUPER EARTH EXOPLANET IN THE HABITABLE ZONE OF A G2 STAR, pp. 1-4,doi:10.5260/cca.199522.

[9] Garner, Rob. "Goldilocks Stars Are Best Places to Look for Life." NASA, NASA, 7 Jan. www.nasa.gov/feature/goddard/2020/goldilocks-stars are-best-places-to-look-for-life.

[10]Gilster, Paul. "Probing Exoplanet Obliquity." Centauri Dreams Imagining and Planning Interstellar Exploration, 2 July 2018, www.centauri dreams.org/2018/07/02/probing-exoplanet-obliquity/.

[11] Hinkel, Natalie. "Stellar Abundances." Stellar Characterization Necessary to Define Holistic Planetary Habitability, arxiv.org/pdf/1904.01089.pdf.

[12] Johnson, Michele. "NASA's Kepler Mission Discovers Bigger, Older Cousin to Earth." NASA, NASA, 23 July 2015, www.nasa.gov/press-release/nasa

kepler-mission-discovers-bigger-older-cousin-to-earth.

[13] Kaib, Nathan A. Galactic Effects on Habitability, 2018, pp. 1-6.

[14] Kane, Stephen R., et al. "Venus as a Nearby Exoplanetary Laboratory." ArXiv.org, 5 Aug. 2020, arxiv.org/abs/2008.01888.

[15] Kopparapu, Ravi Kumar, et al. "STELLAR CHARACTERISTICS FOR HABITABILITY." Characterizing Exoplanet Habitability, arxiv.org/pdf/1911.04441.pdf.

[16] LIBRARY, Spencer Sutton/SCIENCE PHOTO. "Earth's Evolution, Illustration - Stock Image - C029/5733." Science Photo Library, www.sciencephoto.com/media

1730912/view/earth-s-evolution-illustration.

[17] Powell, Devin. "The Drake Equation Revisited: Interview with Planet Hunter Sara Seager." Space.com, Space, 4 Sept. 2013, www.space.com/22648-

drake-equation-alien-life-seager.html.

[18] Wenz, John. "Study Casts Doubt on Existence of a Potential 'Earth 2.0." Scientific American, Scientific American, 9 Apr. 2018, www.scientificamerican.com

/article/study-casts-doubt-on-existence-of-a-potential-earth-2-0/.
[19] "Are We Alone in the Universe? Revisiting the Drake Equation." NASA, NASA, $20 \quad$ May 2016 , exoplanets.nasa.gov/news/1350/are-we-alone-in-the

universe-revisiting-the-drake-equation/.

[20]“Drake Equation." Wikipedia, Wikimedia Foundation, 14 Jan. 2021, en.wikipedia.org/wiki/Drakeequation.

[21] "Formation and Evolution of the Solar System." Wikipedia, Wikimedia Foundation, 9 Jan. 2021 , en.wikipedia.org/wiki/FormationandevolutionoftheSolarSystem.

[22] "Goldilocks Stars: Just Right for Habitable Planets." EarthSky, earthsky.org/space/k-stars-goldilocks-stars-ideal-for-habitable-planets::text $=$ Which $\% 20$ stars $\% 20$ are $\% 20$ the $\% 20$ most, the $\% 20$ sweet $\% 20$ spot $\% 20$ for $\% 2$ Olife.

[23] "History of Earth." Wikipedia, Wikimedia Foundation, 14 Jan. 2021, en.wikipedia.org/wiki/HistoryofEarth

Earth\%20formed\%20around\%204.54\%20billion

atmosphere $\% 20$ contained $\% 20$ almost $\% 20$ no\%20 oxygen.

[24] "Kepler-452b." Encyclopædia Britannica, Encyclopædia Britannica, Inc., www.britannica.com/place/Kepler-452b.

[25] "Kepler-452b." Wikipedia, Wikimedia Foundation, 29 Jan. 2021, en.wikipedia.org/wiki/Kepler-452b.

[26]"Methods of Detecting Exoplanets." Wikipedia, Wikimedia Foundation, 29 Jan. 2021,en.wikipedia.org/wiki/MethodsofdetectingexoplanetsHistory. [27] "Sunspot." Wikipedia, Wikimedia Foundation, 31 Dec. 2020, en.wikipedia.org/wiki/Sunspot.

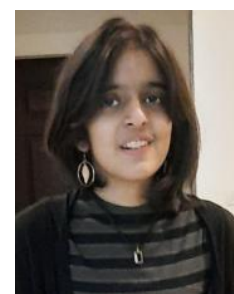

Author--Navya Sahay: High-school student, educated at Neev Academy.

Mentor-- SanahBhimani: Yale Ph.D Candidate 